\title{
BMJ Open Cross-sectional study of the hospital management of adult patients with a suspected seizure (EPIC2)
}

\author{
Jon Mark Dickson, ${ }^{1}$ Hannah Dudhill, ${ }^{2}$ Jane Shewan, ${ }^{3}$ Sue Mason, ${ }^{4}$ \\ Richard A Grünewald, ${ }^{5}$ Markus Reuber ${ }^{6}$
}

To cite: Dickson JM, Dudhill $\mathrm{H}$, Shewan J, et al. Cross-sectional study of the hospital management of adult patients with a suspected seizure (EPIC2). BMJ Open 2017;7:e015696. doi:10.1136/ bmjopen-2016-015696

- Prepublication history and additional material are available. To view these files, please visit the journal online (http://dx.doi.org/ 10.1136/ bmjopen-2016-015696)

Received 3 January 2017 Revised 7 April 2017 Accepted 7 April 2017

\section{(a) CrossMark}

${ }^{1}$ The Academic Unit of Primary Medical Care, The Medical School, Sheffield, England ${ }^{2}$ Sheffield Medical School, The University of Sheffield, Sheffield, South Yorkshire, UK

${ }^{3}$ Research and Development, Yorkshire Ambulance Service NHS Trust, Wakefield, UK ${ }^{4}$ School of Health and Related Research, The University of Sheffield, Sheffield, UK ${ }^{5}$ Department of Neurology, Sheffield Teaching Hospitals NHS Foundation Trust, Sheffield, UK

${ }^{6}$ Academic Neurology Unit, The University of Sheffield, Sheffield, UK

Correspondence to

Dr Jon Mark Dickson;

j.m.dickson@sheffield.ac.uk

\section{ABSTRACT}

Objective To determine the clinical characteristics, management and outcomes of patients taken to hospital by emergency ambulance after a suspected seizure. Design Quantitative cross-sectional retrospective study of a consecutive series of patients.

Setting An acute hospital trust in a large city in England. Participants In 2012-2013, the regions' ambulance service managed 605481 emergency incidents, $74141 / 605481$ originated from Sheffield (a large city in the region), $2121 / 74141$ (2.9\%) were suspected seizures and 178/2121 occurred in May 2012. We undertook detailed analysis of the medical records of the 91/178 patients who were transported to the city's acute hospital. After undertaking a retrospective review of the medical records, the best available aetiological explanation for the seizures was determined.

Results The best available aetiological explanation for $74.7 \%(68 / 91)$ of the incidents was an epileptic seizure, $11.0 \%$ (10/91) were psychogenic non-epileptic seizures and $9.9 \%(9 / 91)$ were cardiogenic events. The epileptic seizures fall into the following four categories: first epileptic seizure $(13.2 \%, 12 / 91)$, epileptic seizure with a historical diagnosis of epilepsy $(30.8 \%, 28 / 91)$, recurrent epileptic seizures without a historical diagnosis of epilepsy $(20.9 \%, 19 / 91)$ and acute symptomatic seizures $(9.9 \%$, 9/91). Of those with seizures (excluding cardiogenic events), $2.4 \%$ (2/82) of patients were seizing on arrival in the Emergency Department (ED), 19.5\% (16/82) were postictal and $69.5 \%(57 / 82)$ were alert. $63.4 \%(52 / 82)$ were discharged at the end of their ED attendance and $36.5 \%$ (19/52) of these had no referral or follow-up. Conclusions Most suspected seizures are epileptic seizures but this is a diagnostically heterogeneous group. Only a small minority of patients require emergency medical care but most are transported to hospital. Few patients receive expert review and many are discharged home without referral to a specialist leaving them at risk of further seizures and the associated morbidity, mortality and health services costs of poorly controlled epilepsy.

\section{INTRODUCTION}

\section{Background}

Epilepsy is an ambulatory care sensitive condition (ACSC) ${ }^{1}$ and suboptimal ambulatory care (also known as routine or scheduled care) leads to unnecessary demand for

\section{Strengths and limitations of this study}

- This is the first study to describe the clinical characteristics, management and outcomes of patients taken to hospital by emergency ambulance after a suspected seizure. The themes identified in this paper resonate with previous studies reflecting practice across the world.

- The triangulation of data drawn from pre-hospital records and hospital medical records (Emergency Department (ED), inpatient and epilepsy clinic) resulted in richer and more robust data than data drawn from a single source.

- Our sample was small and data extraction could have been given added rigour by involving a second rater. Manual data extraction from clinical notes is time consuming which constrained our study methods and is the major limitation to the study.

- Although this study was conducted in a single hospital trust, it is one of the largest acute care providers in the United Kingdom and its ED provides emergency services for a large socioeconomically mixed population.

emergency care. ${ }^{2}$ The majority of epileptic seizures do not require emergency treatment but ambulance services are often called. Calls are rapidly triaged by specialised call handlers, an emergency response vehicle is usually dispatched but, by the time the ambulance arrives most seizures and have terminated spontaneously, nevertheless, the majority of patients are transported to hospital. ${ }^{34}$ Precise estimates vary, but in England (population: 52.96 million, 42.96 million adults), seizures give rise to approximately 211000 calls to ambulance services per year $(3.3 \%$ of all emergency calls). ${ }^{5}$ It is estimated that there are 60000 seizure-related Emergency Department (ED) attendances per year $(2 \%-3 \%$ of all attendances) ${ }^{6}$ and 40000 hospital admissions which represent $9.5 \%$ of all admissions for ACSCs. ${ }^{67}$ There are currently no published studies of care pathways for people who have presented as an emergency with a suspected 
seizure which aim to facilitate urgent medical review ${ }^{8}$ and avoid unnecessary transport to hospital. ${ }^{9}{ }^{10}$ Clinical guidelines for paramedics provide little guidance on the community management and/or referral of patients after a seizure and focus almost exclusively on medical emergencies which are rare. ${ }^{11}$

An epileptic seizure may be the result of suboptimal treatment and should lead to consideration of whether specialist review is required. However, this opportunity to prevent further seizures and/or to refine the patients' emergency care plan is often missed ${ }^{681213}$ Many patients therefore unnecessarily remain at risk of further seizures and the associated morbidity, ${ }^{14}$ mortality ${ }^{15}$ and health services $\operatorname{costs}^{2}{ }^{16}$ of poorly controlled epilepsy. Approximately $70 \%$ of people with epilepsy could become seizure free $\left(\geq 12\right.$ months) with optimal treatment, ${ }^{17-19}$ but internationally, actual seizure freedom rates are significantly lower than this. There is little published data on seizure freedom rates in individual countries ${ }^{1720} 21$ and there are no published international comparisons of seizure freedom rates. The overall seizure freedom rate in the UK is thought to be $50 \% .{ }^{22-25}$ Some epilepsy services in the UK are world-leading but the quality of care is highly geographically variable, and patients in many areas do not have access to optimal monitoring and treatment. This means that as many as one-in-five patients with epilepsy may be unnecessarily having seizures. ${ }^{17}$

\section{Epilepsy Pre-Hospital Interventions and Care Study}

The Epilepsy Pre-Hospital Interventions and Care (EPIC) study was designed to generate data to support improvements in emergency care after a suspected seizure. Despite its importance, this aspect of epilepsy care has received relatively scant academic attention to date. In EPIC1 (5), we described the pre-hospital management of a series of consecutive incidents with suspected seizures. The present study, EPIC2, focusses on the subgroup of these patients that was transported to hospital after a suspected seizure with the aim of determining their clinical characteristics, their management and their outcomes based on data collected from ED, inpatient wards and the epilepsy clinic. The emergency care structure in the UK, with its universal access to healthcare, unitary emergency call handling service and non-overlapping ambulance service and ED provisions, offers opportunities to researchers to study emergency presentations with seizures which do not exist in many other countries.

\section{Methods}

Local context and patient selection

Yorkshire Ambulance Service (YAS) is a regional ambulance service in England (one of the four devolved nations of the UK) covering $9656 \mathrm{~km}^{2}$ and it is the sole provider of ambulance and paramedic services for its population of 4019610 adults (4954876 adults and children) ${ }^{26}$ Sheffield is one of the major urban centres within the area served by YAS and has a population of 451100 adults (551 756 adults and children) which is served by a single hospital-based ED for adults at the Northern General
Hospital site of the Sheffield Teaching Hospitals NHS Foundation Trust (STH) ${ }^{27}$

\section{Case ascertainment, exclusions and missing data}

Patients were retrospectively identified from the records of the YAS. Between 1 April 2012 and 31 March 2013, the YAS dealt with 605481 emergency incidents in adults ( $\geq 16$ years old). Of these incidents, 19799 (3.3\%) were suspected seizures, 2121 originated from Sheffield (a large city in the region) and 178 occurred in May 2012. We analysed data from a sample month, May 2012, which was chosen after preliminary analysis of the summary statistics showed it to be a typical month. ${ }^{5}$ After non-seizure diagnoses and other exclusions were removed, 132 incidents were analysed in detail and 98 were transported to hospital. The initial call handling and out-of-hospital management of these patient was the focus of EPIC1 (5).

Of the 98 incidents from EPIC1 that were transported to hospital, 4/98 incidents were transported to an ED outside Sheffield and 3/98 patients were not identifiable on the STH's computer system so medical records were available for $91 / 98$ incidents. The focus of this paper is the analysis of these 91 incidents although 8/91 (8.8\%) incidents were given non-seizure diagnoses in ED so no further data was collected for these. Detailed data extraction was undertaken for the remaining 83 incidents. The care pathway from emergency call to discharge from hospital including exclusions is complex. It is summarised in figure 1.

\section{Data collection and analysis}

Data were extracted by one of the authors (HD) using a data extraction tool (see online supplementary file) which was developed by all the authors and was revised after an initial pilot. Some variables such as the working diagnosis changed throughout the care pathway and so we report the results separately at each stage in the pathway: (1) ED, (2) inpatient wards, (3) outpatient epilepsy clinic and (4) combined data from all three sources. The data presented in section 4 was drawn together by HD from all available sources (ED notes, inpatient notes, epilepsy clinic notes) to document an overview of the hospital management of each incident taking into account the opinion of all the clinicians involved throughout the care pathway. This allowed triangulation of the data, which allowed resolution of inconsistencies between, for example, accounts in the ED notes and in the epilepsy clinic notes, and it allowed us to draw robust conclusions about the best available aetiological explanation for the index event. If the best available aetiological explanation for the suspected seizure was an epileptic seizure, it allowed us to determine if the patient had a historical diagnosis of epilepsy.

In this paper, we report the data as it was recorded in the notes by the clinicians involved in the incidents with as little interpretation from the authors as possible. Where interpretation was required, we included definitions within the data collection tool to inform these judgements; these are described below. We analysed each incident separately 


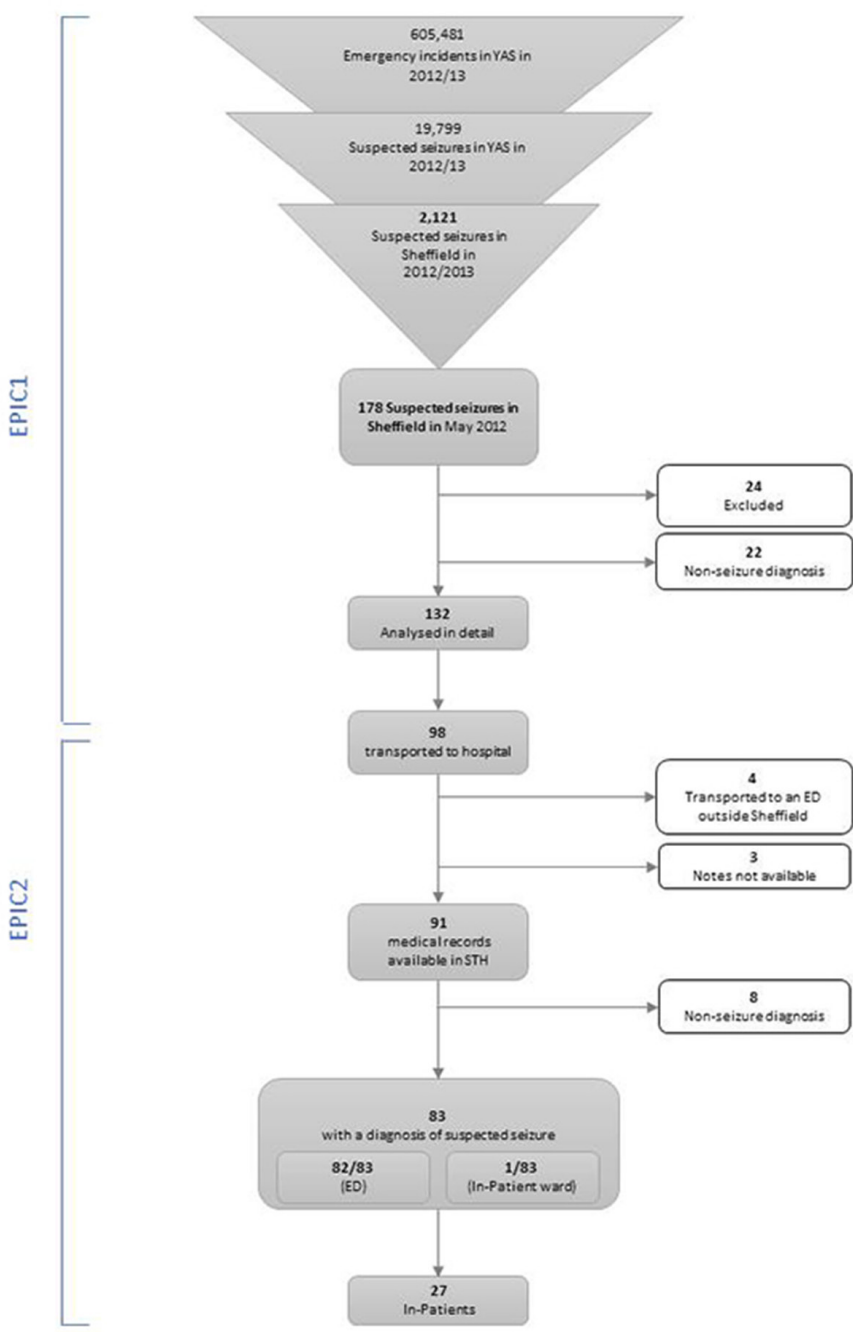

Figure 1 Flow chart to illustrate the care pathway and exclusions throughout the EPIC study (EPIC1 and EPIC2). The EPIC1 exclusions were as follows: missing/inadequate data (18/178) and miscellaneous, for example, hoax call (6/178). The non-seizure diagnoses in EPIC1 were as follows: syncope, ${ }^{3}$ intoxicated/passed out, ${ }^{2}$ tremor/spasm, ${ }^{2}$ fall, ${ }^{2}$ rigours, ${ }^{2}$ twitching, ${ }^{1}$ panic attack, ${ }^{1}$ anxiety/hyperventilation, ${ }^{2}$ abnormal behaviour ${ }^{1}$ and social/miscellaneous/ inappropriate. ${ }^{6}$ The non-seizure diagnoses in EPIC2 were 5/8 vasovagal, $1 / 8$ syncope, $1 / 8$ complete heart block and 1/8 collapse. EPIC, Epilepsy Pre-Hospital Interventions and Care.

(some patients attended more than once during the study period and therefore generated multiple incidents). To calculate the number of incidents in which all physiological parameters were normal, we used the following parameters: heart rate $(60-100 \mathrm{bpm})$, respiratory rate (14-18 breaths per minute), systolic blood pressure $(100-140 \mathrm{~mm} \mathrm{Hg})$, blood glucose $(3.5-11.1 \mathrm{mmol} / \mathrm{L})$, temperature $\left(36.5^{\circ} \mathrm{C}-37.5^{\circ} \mathrm{C}\right), \mathrm{O}_{2}$ saturations $(<94 \%)$ and Glasgow Coma Score (GCS) $(15 / 15)$. We excluded low temperature $\left(<36.5^{\circ} \mathrm{C}\right)$ from this calculation because it is very unlikely that any patients truly had hypothermia in May in England (a low temperature measurement is likely to reflect inaccuracy of peripheral temperature recording). We recorded the Sheffield Early Warning
Score (SHEWS) which is based on the National Early Warning Score. SHEWS is a composite score based on heart rate, respiratory rate, oxygen saturation, systolic blood pressure, urine output and consciousness level. A score above 0 identifies patients who may be acutely ill so that their care can be stepped-up: $1=$ increase frequency of observations and inform nurse, $2=$ hourly observations and consider medical review, $3=$ immediate medical review. Numerical data were entered into SPSS which was used for calculating summary statistics. $\chi^{2}$ tests were used to assess relationships between categorical variables. Where there was missing data, it was coded as such in SPSS. Variables with large numbers of missing data points were excluded from the analysis and are not reported in the Results. Small numbers of missing data points in specific variables are not reported in the Results.

\section{Definitions}

The latest International League Against Epilepsy definitions of epilepsy and seizure ${ }^{28} 29$ were applied during data collection. We categorised the indication for admission: medical (seizure-related), medical (not seizure-related) and social. Medical (seizure-related) admissions were those where the principal reason for admission was seizure(s), for example, patients who were still postictal at the end of their ED attendance. Medical (not seizure-related) admissions were those where the principal reason for admission was not a seizure, for example, lower respiratory tract infection or unexplained fever. Social admissions were those where there was no medical reason for admission but it was not possible to discharge the patient directly from the ED, for example, because a home care package had to be put in place. We categorised those with and without a self-reported diagnosis of epilepsy prior to the index event. Historical diagnoses of epilepsy were determined from the records of the ED doctors and often only brief information was available. In addition to the seizure, we determined if the patient had any other acute clinical problems. Alcohol was deemed to be a clinical problem if there was documentation of excessive alcohol ingestion which occurred around the time of the index event or if there was a history of alcohol abuse (such as alcohol dependency or withdrawal seizures). Patients were deemed to have recovered fully if they were recorded as having returned to their normal level of functioning with no acute medical problems that required hospital assessment and/or treatment and were not thought to be postictal (notwithstanding tiredness, headache and myalgia).

\section{RESULTS}

\section{Demographics and repeat attendances}

The patients' median age was 40 years (IQR, 30; range, 16-97). Males accounted for $54.2 \%$ of incidents. The 83 incidents relate to 79 patients. A total of $4 / 79$ (5.1\%) patients generated two incidents during the 1-month study period. The time intervals between the repeat 


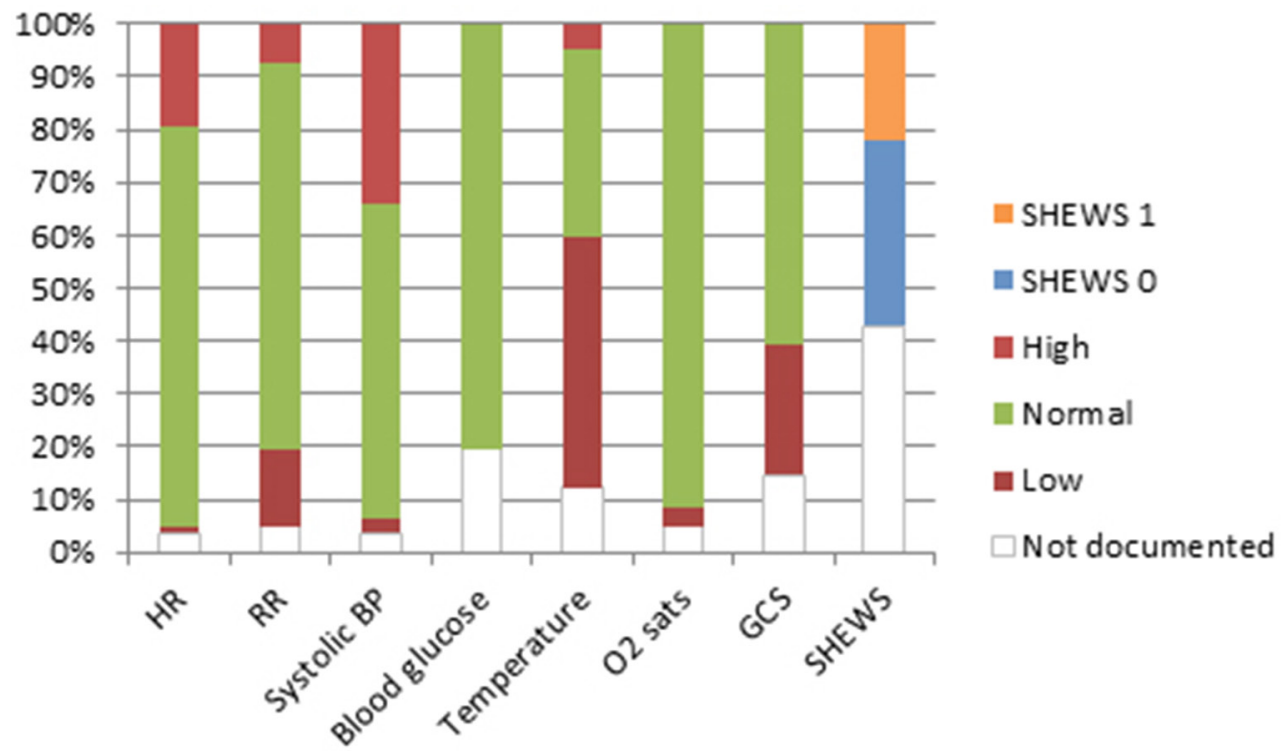

Figure 2 Physiological parameters and the Sheffield Early Warning Score (SHEWS) for each of the patients on arrival in the Emergency Department. SHEWS score: 1 = increase frequency of observations and inform nurse, $2=$ hourly observations and consider medical review, 3 = immediate medical review. No patients had a SHEWS score recorded that was higher than 1. Normal ranges: HR (60-100 bpm), RR (14-18 breaths per minute), systolic BP (100-140 mm Hg), blood glucose (3.5$11.1 \mathrm{mmol} / \mathrm{L})$, temperature $\left(36.5^{\circ} \mathrm{C}-37.5^{\circ} \mathrm{C}\right), \mathrm{O}_{2}$ sat $(<94 \%)$ and GCS (15/15). BP, blood pressure; GCS, Glasgow Coma Score; $\mathrm{O}_{2}$ sats, oxygen saturations; $\mathrm{HR}$, heart rate; $\mathrm{RR}$, respiratory rate.

attendances were as follows: 1 day, 3 days, 13 days and 15 days.

\section{Management in the ED \\ Medical history}

A total of $82 / 83$ incidents were seen in ED, one incident was admitted directly to a neurology ward (without being seen in ED). The ED records documented a history of a seizure disorder in $43.9 \%(36 / 82)$ of all incidents: epilepsy, 36.6\% (30/82); psychogenic non-epileptic seizures (PNES), 6.1\% (5/82); and epilepsy plus PNES, $1.2 \%(1 / 82)$.

\section{Clinical details}

In $2.4 \%(2 / 82)$ of incidents, the patient was seizing on arrival at the ED, $69.5 \%(57 / 82)$ were alert on arrival, $19.5 \%(16 / 82)$ were postictal and, in $6 / 82(7.3 \%)$, their status was not clear from the notes. All physiological parameters were normal (or not recorded) in 28/82 $(34.1 \%)$ of incidents but often only one parameter was abnormal and often this was mild tachycardia or mild hypertension (see figure 2). In the incidents in which the seizure had terminated prior to arrival in the ED, $76.25 \%$ $(61 / 80)$ had no subsequent seizures during their hospital stay. A total of $17.5 \%(14 / 80)$ had recurrent seizures either in the ED or after transfer to a hospital ward. In $7.3 \%(6 / 82)$ of incidents, emergency medication for the termination of seizures was administered in the ED. Of cases presented, $70.7 \%(58 / 82)$ had recovered fully by the end of the ED attendance.

Final diagnosis at the end of the ED attendance

In the opinion of the ED clinicians, 63/82 incidents were likely to have been epileptic seizures: $13.4 \%$ (11/82) had experienced a first fit, 35.4\% (29/82) had experienced an epileptic seizure in the context of an historical diagnosis of epilepsy and $28.0 \%(23 / 82)$ had experienced an epileptic seizure with a history of recurrent seizures but without an historical diagnosis of epilepsy. A total of $7.3 \%(6 / 82)$ were diagnosed with an acute symptomatic seizure. A total of $9.8 \%(8 / 82)$ were diagnosed with a PNES. The diagnosis was unknown in $2.4 \%(2 / 82)$, and in $3.7 \%(3 / 82)$, no diagnosis was recorded.

\section{Other clinical problems}

Of all cases presented, $47.6 \%(39 / 82)$ had another clinical problem(s) in addition to the suspected seizure. This was sometimes secondary to the suspected seizure but was often unrelated or only tangentially related to it. $35.4 \%$ $(29 / 82)$ had one additional clinical problem, $8.5 \%$ $(7 / 82)$ had two, $2.4 \%(2 / 82)$ had three and $1.2 \%(1 / 82)$ had four. In total, there were 53 additional clinical problems among the 82 incidents. Of these, $41.5 \%(22 / 53)$ were alcohol, $15.0 \%(8 / 53)$ were injuries, $9.4 \%(5 / 53)$ were illicit drug use and $34.0 \%(18 / 53)$ were 'other' (eg, brain tumour, fever, acidosis, psychiatric problems and abdominal pain).

\section{Discharge and referral from ED}

Of all patients, $63.4 \%(52 / 82)$ were discharged home at the end of their ED attendance, 31.7\% (26/82) were admitted to an inpatient ward from ED (another patient who was taken directly to a ward by ambulance without attending ED) and $4.9 \%$ (4/82) self-discharged. The indication for admission was related to the seizure in $61.5 \%(16 / 26)$ of cases. In $26.9 \%(7 / 26)$ of cases, it was due to a medical problem which was not obviously related to the seizure (eg, chest infection, gastrointestinal problems, infective rash), and in $11.5 \%$ (3/26) of cases, 
the reason for admission was social. Of the patients who were discharged home from the ED, only $61.5 \%(32 / 52)$ had documented referral or follow-up advice. A total of $34.6 \%(18 / 52)$ were referred to an epilepsy clinic, $25.0 \%$ $(13 / 52)$ were referred to their general practitioner and $1.9 \%(1 / 52)$ were referred to an epilepsy specialist nurse. A total of $36.5 \%$ (19/52) had no documented referral, and $52.6 \%(10 / 19)$ of these had not been seen in the epilepsy clinic before the event. There was a relationship between SHEWS and GCS on arrival in ED with disposal (admission or discharge). Patients with an abnormal SHEWS on arrival were more likely to be admitted to hospital and less likely to be discharged $(\chi 2(1, \mathrm{n}=45)=10.385, \mathrm{p}=0.001)$, likewise for patients with a reduced GCS on arrival $(\chi 2$ (1, $\mathrm{n}=68)=15.451, \mathrm{p}=0.000085$ ).

\section{Inpatient management}

\section{Length of stay and specialty}

A total of 27 patients were admitted to an inpatient medical ward. The median duration of admission was 2.0 days (IQR, 5.0; range, 0-17). 66.7\% (18/27) were admitted to a general medical ward, $18.5 \%(5 / 27)$ were admitted under neurology, $7.4 \%$ (2/27) were admitted to the intensive care unit and the remaining $7.4 \%(2 / 27)$ were admitted under other specialities (infectious diseases and the surgical admissions centre). 29.6\% (8/27) were transferred to another specialty during their admission (four to gastroenterology, two to neurology and two to general medicine).

\section{Final diagnosis at the end of the inpatient admission}

Of those that were diagnosed with an epileptic seizure, $44.4 \%(12 / 27)$ had an historical diagnosis of epilepsy, $22.2 \%(6 / 27)$ had a history of recurrent seizures without an historical diagnosis of epilepsy and none had experienced a first fit. $14.8 \%(4 / 27)$ were diagnosed with an acute symptomatic seizure and $7.4 \%(2 / 27)$ were diagnosed with a non-epileptic attack.

\section{Discharge and referral}

$66.7 \%(18 / 27)$ were eventually discharged home from the hospital ward. All 18 were fully recovered at discharge. $11.1 \%(3 / 27)$ self-discharged without waiting for medical assessment. Of the patients who were discharged, 33.3\% $(6 / 18)$ had no documented referral or follow-up. A total of $44.4 \%(8 / 18)$ were referred to an epilepsy clinic and $11.1 \%(2 / 18)$ were referred to an epilepsy specialist nurse. Two out of six $(33.3 \%)$ patients with no referral were already under the care of the epilepsy clinic and may have had direct access to their services making formal referral unnecessary and one patient $(1 / 18,5.6 \%)$ already had an epilepsy clinic appointment scheduled for the next day.

\section{Epilepsy clinic data}

Of all patients with a suspected seizure who were transported to the ED during the study period, 63.4\% (52/82) had been seen in the only specialist epilepsy clinic in the city. Of these, $51.9 \%(27 / 52)$ had been seen before the index event and 25/52 (48.1\%) had been seen after the index event. In $75.0 \%(39 / 52)$ of these patients, a diagnosis of epilepsy was made in the clinic (either before or after the index event). In $66.7 \%$ (26/39), the diagnosis was localisation-related epilepsy; in $17.9 \%(7 / 39)$, it was generalised epilepsy. In $15.4 \%$ (6/39), the epilepsy type was undetermined. In $15.4 \%$ (8/52), a diagnosis of PNES was made in the epilepsy clinic.

\section{Data from all sources}

\section{Epilepsy medication}

$38.6 \%(32 / 83)$ had a documented history of anti-epileptic drug (AED) use at the time of the index event: $56.3 \%$ (18/32) were receiving mono-therapy and $43.8 \%$ (14/32) poly-therapy. A suspicion of non-compliance with medication was documented in $18.8 \%(6 / 32)$.

\section{General management and seizure-related injuries}

AED regimes were changed or AEDs started during the hospital attendance/admission in $16.9 \%(14 / 83)$ of cases (in the ED or on the wards). Excluding the treatment of injuries, $36.1 \%(30 / 83)$ received some form of acute medical treatment (including complex treatments, such as for alcohol withdrawal, as well as relatively minor treatments such as pain relief with paracetamol or codeine). Of the incidents reported, $7.2 \%(6 / 83)$ sustained an injury but only $1.2 \%(1 / 83)$ received major treatment (defined as that which probably required an acute hospital) for their injury (shoulder dislocation) and $2.4 \%(2 / 83)$ received minor treatment (defined as that which probably could have been delivered elsewhere) (wound care and treatment for an avulsed toenail). Although there was no tangible intervention in many incidents, all the patients received monitoring, assessment and diagnosis from a doctor and/or other clinicians.

\section{Investigations}

$22.9 \%(19 / 83)$ had neuroimaging at some point during their attendance or admission. No patients had an EEG performed in the ED or as an inpatient. EEG tests, if considered necessary, were carried out as routine outpatient tests after the patient's discharge from their emergency care episode.

\section{Seizure recurrence}

In $73.5 \%(61 / 83)$ of incidents, the index event was the only seizure the patient had experienced in the 24 hours prior to their arrival in the ED. 21.7\% (18/83) had experienced at least one other seizure in the previous 24 hours ('recurrent seizures'). Of those admitted after a single seizure, $14.8 \%(9 / 61)$ went on to have a subsequent seizure(s) in hospital. Of those with recurrent seizures, $27.8 \%(5 / 18)$ went on to have subsequent seizures in hospital. A $\chi^{2}$ test of independence was performed to examine the relationship between seizure presentation on arrival (single or recurrent) and seizure recurrence during the hospital stay. The relationship was not significant, $\chi^{2}(1, \mathrm{n}=83)=1.38, \mathrm{p}=0.24$. Of all patients, $10.8 \%$ $(9 / 83)$ had emergency medication administered by either a carer or ambulance crew before arrival in the ED. Of these, $77.8 \%(7 / 9)$ were either still seizing on arrival 

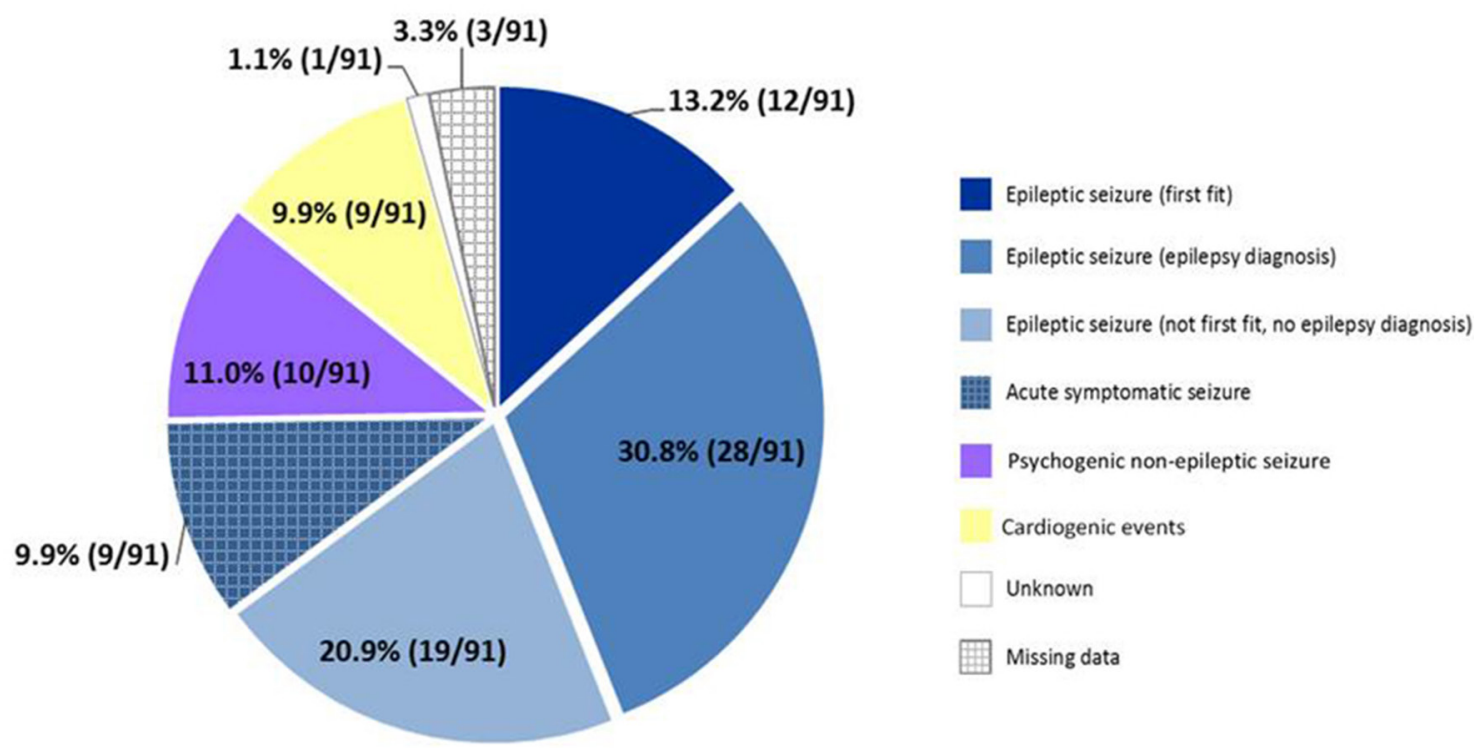

Figure 3 Best available aetiological explanation for the index event. Acute symptomatic causes were: alcohol withdrawal, ${ }^{6}$ head injury, ${ }_{1}$ hypoglycaemia ${ }^{1}$ and transient ischaemic attack. ${ }^{1}$ The cardiogenic events were vasovagal episode,${ }^{6}$ syncope, ${ }^{1}$ complete heart block ${ }^{1}$ and collapse. ${ }^{1}$

in the ED or went on to have recurrent seizures during their hospital attendance.

Best available aetiological explanation for the index event The majority of suspected seizures in EPIC2 were epileptic $(68 / 91(74.7 \%)$ (including acute symptomatic seizures)) but only 28/91 (30.8\%) had a diagnosis of epilepsy. The epileptic seizures fall into the following four categories: a first epileptic seizure $(13.2 \%, 12 / 91)$, an epileptic seizure in a patient with a historical diagnosis of epilepsy $(30.8 \%$, 28/91), an epileptic seizure in a patient known to have recurrent seizures but without a historical diagnosis of epilepsy $(20.9 \%, 19 / 91)$ and acute symptomatic seizures in $9.9 \%(9 / 91)$. The other two important diagnostic categories were PNES 11.0\% (10/91) and cardiogenic events $9.9 \%(9 / 91)$. The best available aetiological explanation for the index event is summarised in figures 3 and 4 shows diagnoses at each stage in the care pathway.

\section{Diagnostic accuracy in the ED}

Of all diagnoses recorded at the time of initial ED admission, $87.8 \%(72 / 82)$ concurred with diagnoses made after more specialist or prolonged assessment during an inpatient stay or in a specialist in clinic. In those with an ED diagnosis of epileptic seizure, 98.3\% (58/59) had a concordant diagnosis after inpatient admission or more specialist review.

\section{DISCUSSION}

\section{Aetiology and significance of emergency calls for suspected} seizures

This is the first study to quantify diagnoses among pre-hospital patients after a suspected seizure. Our data show that $74.7 \%$ of our patients had suffered an epileptic seizure, $11.0 \%$ had suffered a PNES and 9.9\% had suffered a cardiogenic event (see figure 3). The patients with epilepsy fall into three subgroups: epileptic seizure (first fit), epileptic seizure (epilepsy diagnosis) and epileptic seizure (not first fit, no epilepsy diagnosis). Although many of these patients did not require emergency treatment, patients in all three groups could benefit from a review in the next few days by an epilepsy specialist after a seizure ${ }^{8}$ (this also applies to the patients with PNES). Despite this, our data is consistent with other large studies ${ }^{6}$ which show that most patients are discharged without the input of an epilepsy specialist or follow-up. This leaves all these patients (not just those with epilepsy and PNES) at risk of recurrence and the associated morbidity, mortality and health services costs of these events. ${ }^{2}$

\section{Medical emergencies and clinical risk management}

A large majority of the patients in our study were not acutely unwell on arrival at hospital. These results are consistent with qualitative data suggesting that major factors in deciding to call for an emergency ambulance and transporting patients to hospital after a suspected seizure are lack of confidence and medico-legal concerns among patients, carers, the public and paramedics rather than true clinical need. ${ }^{34}{ }^{30}$ We were unable to define the exact proportion of patients that were potentially suitable for community management without transport to hospital or discharge from ED. This would require a criterion-based approach and further research would be required to define criteria which can be used to identify patients suitable for non-transport and how to overcome barriers to community management such as the presence of other clinical problems, risk stratification for recurrence of seizures, appropriate levels of supervision and safe management of the postictal phase.

The risk of seizure recurrence and the phenomenon of seizure clusters are a major factor in management decisions by clinicians but they are poorly understood. ${ }^{31}$ 
A

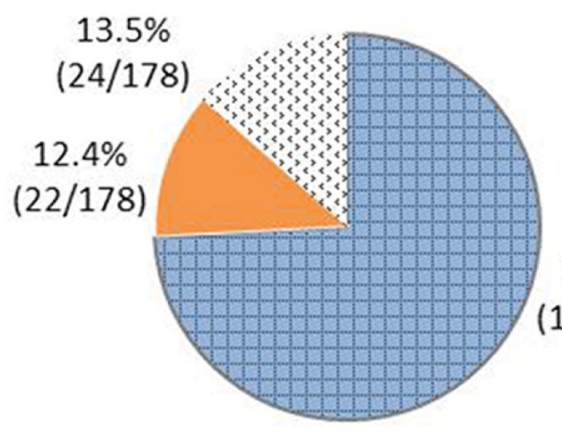

$74.2 \%$

C

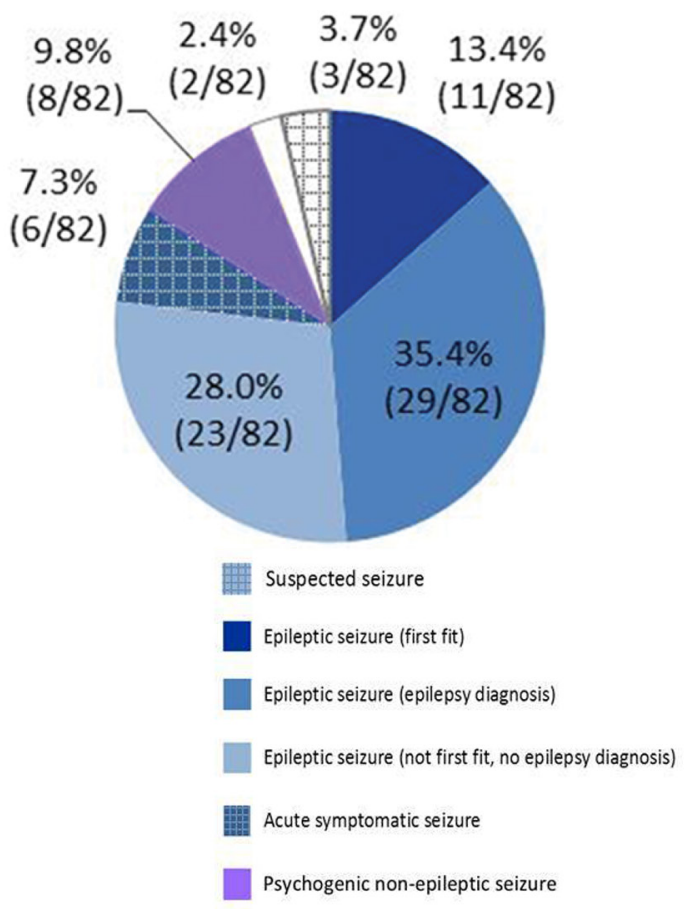

B

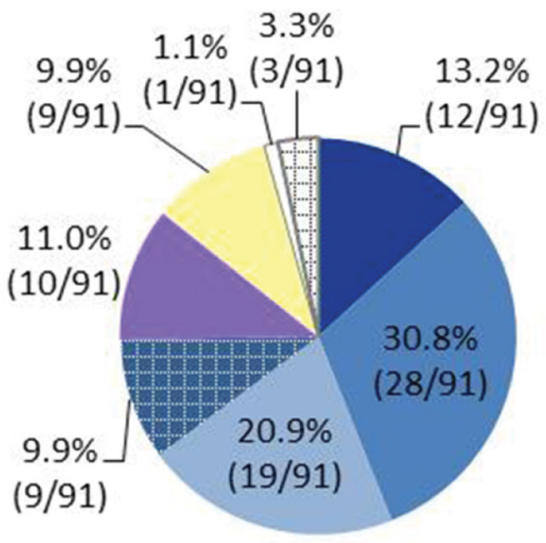

D

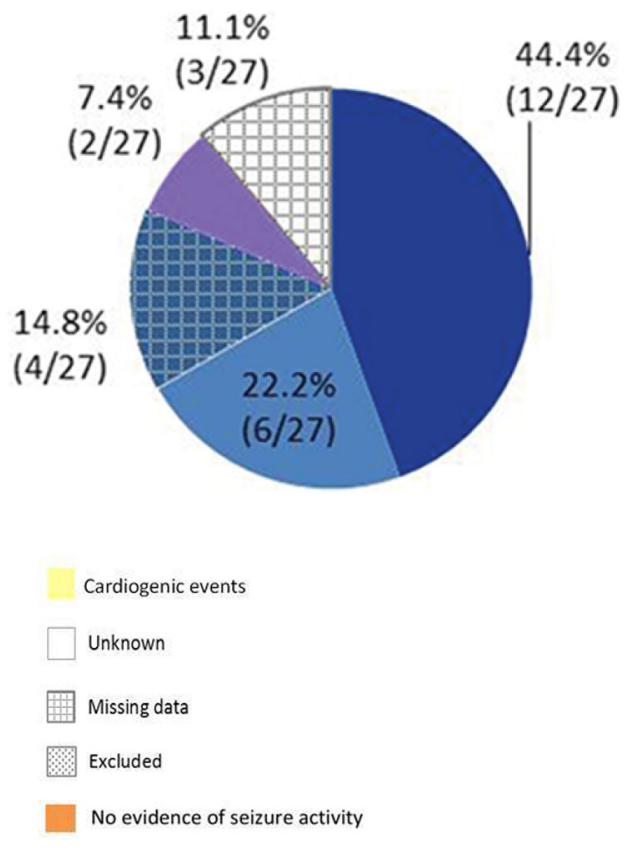

Figure 4A-D Data from EPIC1 and EPIC2 to illustrate diagnoses, exclusions and missing data at each stage in the care pathway. Pre-Hospital Diagnoses(A). In Sheffield May 2012, there were 178 suspected seizures for which 999 was called. A total of 24/178 were excluded and 22/178 were not seizures leaving 132/178 suspected seizure incidents that were studied in detail in EPIC1. Exclusions: missing/inadequate data (18/178, 10.1\%) and miscellaneous, for example, hoax call $(6 / 178,3.4 \%)$. The clinical impression of the ambulance clinicians was that there was no evidence of seizure activity in 22/178 (12.4\%). Not seizure diagnoses: syncope (3), intoxicated/passed out (2), tremor/spasm (2), fall (2), rigours (2), twitching (1), panic attack (1), anxiety/ hyperventilation (2), abnormal behaviour (1) and social/miscellaneous/inappropriate (6). Hospital Diagnoses (B-D). Best Available Diagnoses (B). The hospital notes of 91/132 were analysed in detail (98/132 were transported to hospital but 4/98 transported to an hospital outside Sheffield and 3/98 sets of notes were not available). The best available data for the aetiology of the 91 events is shown in B (this is based on data from all sources: ED notes, inpatient notes and epilepsy clinic notes). Aetiology of acute symptomatic seizures: alcohol withdrawal (6), head injury (1), hypoglycaemia (1) and transient ischaemic attack (1). Emergency Department Diagnoses (C). A total of 82/91 that were transported to ED at Sheffield Teaching Hospitals NHS Foundation Trust were suspected seizures. A total of 9/91 were given non-seizure diagnoses: vasovagal episode (6), syncope (1), complete heart block (1) and collapse (1). NB/An additional 1/91 suspected seizure was transported direct to an inpatient ward so 82/91 were diagnosed with suspected seizures. Inpatient Diagnoses (D). A total of 27/83 were admitted to an inpatient ward.

We are not aware of any prospective studies specifically looking at the short-term risk of seizure recurrence in the community. The published evidence in this area focusses on long-term recurrence risk after a seizure ${ }^{32} 33$ and the treatment of status epilepticus ${ }^{34}$ (defined as ongoing seizure activity or recurrent seizures). Our data showed that short-term recurrence is not more likely in patients who have presented with more than one seizure, 
compared with those who present with a single seizure, but the numbers in each group were small and further research is required.

\section{Non-compliance, alcohol and difficult to reach groups}

Of all patients in our study with recurrent seizures, $20.9 \%$ (19/91) did not have an historical epilepsy diagnosis. This might partly be a reflection of inadequate medical records, and using additional data sources such as general practitioner records may have reduced the number in this category. However, this result may reflect a more substantial problem of unmet need and is consistent with the National Audit of Seizure management in Hospitals (NASH) ${ }^{6}$ a large national audit conducted in the National Health Service in the UK. We did not collect data to analyse this phenomenon further but we did find that alcohol use and illicit drugs were common clinical problems as was non-adherence with AED treatment. Non-adherence is associated with increased seizure frequency, adverse outcomes and increased hospital attendance/admission and higher healthcare costs. ${ }^{35-37}$ These data suggest that these patients may not understand the importance and benefits of medical advice, may be socially isolated and are perhaps living chaotic lifestyles. Simply improving access to medical services may not be an effective solution and more active outreach programmes may be required to reach this group. ${ }^{38}$ Hospital-based alcohol nurses and ambulance service alcohol referral pathways may be able to intervene in these cases and facilitate joint working between alcohol services and services for people with epilepsy.

\section{Demographics, re-attendance and specialist review}

Of our patients, $5.1 \%(4 / 79)$ re-attended within the 1-month study period. This probably under-estimates the true repeat attendance rate because of the short time window. Other studies have estimated this figure as to be as high as $60 \%$ within 1 year. ${ }^{39}$ The age histogram of our cohort was uni-modal with a cut-off at age 16 years (children were excluded) and a peak incidence at age 40 years. However, the age-related incidence curve of epilepsy has two peaks, one in childhood and the other in old age ${ }^{40}$ This inconsistency which has been reported elsewhere $^{41}$ may be explained by underlying seizure frequency in this group but other factors are likely to be more important such as alcohol use and thresholds for accessing care. ${ }^{42}{ }^{43}$ Consistent with the NASH audit, our data has shown suboptimal rates of referral to epilepsy specialists and low rates of intervention such as inpatient specialist review, epilepsy-specific investigations or modification of AEDs. ${ }^{8}$ Follow-up by specialist epilepsy nurses has been shown to be associated with earlier discharge from hospital. ${ }^{39}$ Expansion of the specialist nurse role may be a solution to problems with lack of capacity in some consultant-led services. ${ }^{44}$

\section{CONCLUSIONS}

Suspected seizures generate significant demand for emergency care (pre-hospital and hospital). Most suspected seizures are epileptic and often reflect failed ambulatory care for epilepsy. Emergency calls to ambulance services are an opportunity to improve seizure freedom rates by facilitating urgent review by an epilepsy specialist. Many patients do not require emergency hospital treatment and there is the potential to develop pathways which both avoid unnecessary hospital attendance/admission and facilitate specialist review. The EPIC study (EPIC1 and EPIC2) provides good-quality data to stimulate further research and to conceptualise the reconfiguration of services which aim to maximise seizure freedom rates in people with epilepsy and to prevent avoidable attendances at hospital.

Acknowledgements Many thanks to Trevor Baldwin whose expertise, support and advice were invaluable during the EPIC study.

Contributors The research was suggested by MR and RAG. They provided important advice and comments on the protocol and manuscript throughout. JMD took the lead with the study design, contacting collaborators, submitting ethics applications and other permissions, supervising $\mathrm{HD}$ and writing the manuscript. HD took the lead for data collection, data analysis, presentation and interpretation of the results. She wrote some parts of the manuscript and reviewed the manuscript throughout its preparation by JMD. SM and JS were involved in study design and data collection and provided advice and comments on the protocol and manuscript throughout. JMD and HD are joint first authors and RAG and MR are joint senior authors.

Funding This work was supported by the NIHR CLAHRC Yorkshire and Humber (www.clahrc-yh.nihr.ac.uk) although the project received no specific grant from any funding agency in the public, commercial or not-for-profit sectors. The views expressed are those of the author(s) and not necessarily those of the NHS, the NIHR or the Department of Health.

Competing interests JMD received a grant of $£ 3500$ in 2014 from UCB Pharma Limited (paid to The University of Sheffield) to study unscheduled admissions for seizures using Hospital Episode Statistics. UCB had no direct input into the project. The other authors have no competing interests.

Patient consent Detail has been removed from this case description/these case descriptions to ensure anonymity. The editors and reviewers have seen the detailed information available and are satisfied that the information backs up the case the authors are making.

Ethics approval University of Sheffield Research Ethics Committee.

Provenance and peer review Not commissioned; externally peer reviewed.

Data sharing statement No data sharing agreements are in place but the authors welcome queries from people interested in viewing the original data.

Open Access This is an Open Access article distributed in accordance with the Creative Commons Attribution Non Commercial (CC BY-NC 4.0) license, which permits others to distribute, remix, adapt, build upon this work non-commercially, and license their derivative works on different terms, provided the original work is properly cited and the use is non-commercial. See: http://creativecommons.org/ licenses/by-nc/4.0/

(c) Article author(s) (or their employer(s) unless otherwise stated in the text of the article) 2017. All rights reserved. No commercial use is permitted unless otherwise expressly granted.

\section{REFERENCES}

1. Bardsley M, Blunt I, Davies S, et al. Is secondary preventive care improving? Observational study of 10-year trends in emergency admissions for conditions amenable to ambulatory care. BMJ Open 2013;3:e002007

2. Manjunath R, Paradis PE, Parisé $H$, et al. Burden of uncontrolled epilepsy in patients requiring an emergency room visit or hospitalization. Neurology 2012;79:1908-16. 
3. Burrell L, Noble A, Ridsdale L. Decision-making by ambulance clinicians in London when managing patients with epilepsy: a qualitative study. Emerg Med J 2013;30:236-40.

4. O'Hara R, Johnson M, Siriwardena AN, et al. A qualitative study of systemic influences on paramedic decision making: care transitions and patient safety. J Health Serv Res Policy 2015;20:45-53.

5. Dickson J, Taylor L, Shewan J, et al. Reuber M. A cross-sectional study of the pre-hospital management of adult patients with a suspected seizure (EPIC1). BMJ Open 2016;2015:e010573.

6. Dixon PA, Kirkham JJ, Marson AG, et al. National Audit of Seizure management in Hospitals (NASH): results of the national audit of adult epilepsy in the UK. BMJ Open 2015;5:e007325.

7. Tian Y, Dixon A, Gao H. Emergency hospital admissions for ambulatory care-senstive conditions: identifying the potenetial for reductions. The King's Fund 2012.

8. National Institute of Clinical Excellence. The epilepsies: the diagnosis and management of the epilepsies in adults and children in primary and secondary care. 2012.

9. Scottish Ambulance Service. A Review of Scottish Ambulance Service See and Treat Guidelines. 2007.

10. Scottish Ambulance Service. Treat and Refer Guidelines: Do We Like Them? Do We UseThem? 2007.

11. Snooks HA, Dale J, Hartley-Sharpe C, et al. On-scene alternatives for emergency ambulance crews attending patients who do not need to travel to the accident and emergency department: a review of the literature. Emerg Med J 2004;21:212-5.

12. National Insititute for Health and Care Excellence. Transient loss of consciousness ('blackouts') management in adults and young people. 2010.

13. Malmgren K, Reuber M, Appleton R. Differential diagnosis of epilepsy. Oxford Textbook of Epilepsy and Epileptic Seizures Oxford University Press, 2013.

14. Baker GA, Jacoby A, Buck D, et al. Quality of life of people with epilepsy: a European study. Epilepsia 1997;38:353-62.

15. Lhatoo S, Johnson A, Goodridge D, et al. Mortality in epeilepsy in the first 11 to 14 years after diagnosis: miltivariate analysis of a longterm, prospective, population-based cohort. Annals of Neurology 2001;2001:336-44.

16. Galarraga JE, Mutter R, Pines JM. Costs associated with ambulatory care sensitive conditions across hospital-based settings. Acad Emerg Med 2015;22:172-81.

17. Moran NF, Poole K, Bell G, et al. Epilepsy in the United Kingdom: seizure frequency and severity, anti-epileptic drug utilization and impact on life in 1652 people with epilepsy. Seizure 2004;13:425-33.

18. Sander JW. The use of antiepileptic drugs-principles and practice. Epilepsia 2004;45 Suppl 6:28-34.

19. Kwan P, Brodie MJ. Early identification of refractory epilepsy. $N$ Engl J Med 2000;342:319.

20. Relationship between seizure frequency and costs and quality of life of outpatients with partial epilepsy in France, Germany and the United Kingdom.

21. ILAE Commission on the Burden of Epilepsy, Subcommission on the Economic Burden of Epilepsy: final report. 1998-2001.

22. Association of British Neurologists. Acute neurology services survey. 2014, 2014

23. Dickson JM, Scott PA, Reuber M. Epilepsy service provision in the National Health Service in England in 2012. Seizure 2015;30:26-31.
24. Pearson M, Marson T, Dixon P, et al. National Audit of Seizure management in Hospitals (Clinical Report), 2012.

25. Pearson M, Marson T, Dixon P, et al. National Audit of Seizure management in Hospitals (Clinical Report), 2014.

26. Association of Ambulance Chief Executives. http://aace.org.uk/ukambulance-service/.

27. Office for National Statistics. Annual Mid-year Population Estimates for Clinical Commissioning Groups, Mid-2011. http://www.ons.gov. uk.

28. Fisher RS, van Emde Boas W, Blume W, et al. Epileptic seizures and epilepsy: definitions proposed by the International League against Epilepsy (ILAE) and the International Bureau for Epilepsy (IBE). Epilepsia 2005;46:470-2.

29. Fisher RS, Acevedo C, Arzimanoglou A, et al. ILAE official report: a practical clinical definition of epilepsy. Epilepsia 2014;55:475-82.

30. Ridsdale L, Virdi C, Noble A, et al. Explanations given by people with epilepsy for using emergency medical services: a qualitative study. Epilepsy Behav 2012;25:529-33.

31. Haut SR, Lipton RB, LeValley AJ, et al. Identifying seizure clusters in patients with epilepsy. Neurology 2005;65:1313-5.

32. Hart YM, Sander JWAS, Shorvon SD, et al. National General Practice Study of Epilepsy: recurrence after a first seizure. The Lancet 1990;336:1271-4.

33. Bonnett LJ, Marson AG, Johnson A, et al. External validation of a prognostic model for seizure recurrence following a first unprovoked seizure and implications for driving. PLoS One 2014;9:e99063.

34. Alldredge BK, Gelb AM, Isaacs SM, et al. A comparison of lorazepam, diazepam, and placebo for the treatment of out-ofhospital status epilepticus. N Engl J Med 2001;345:631-7.

35. Manjunath R, Davis KL, Candrilli SD, et al. Association of antiepileptic drug nonadherence with risk of seizures in adults with epilepsy. Epilepsy \& behavior: E\&B 2009;14:372-8.

36. Davis KL, Candrilli SD, Edin HM. Prevalence and cost of nonadherence with antiepileptic drugs in an adult managed care population. Epilepsia 2008;49:446-54.

37. Eea F. Non-adherence to antiepileptic drugs and increased mortality. Neurology 2003.

38. Davis J, Lovegrove M. Inclusion health: education and training for health professionals. Allied Health Solutions Limited, 2015.

39. Noble AJ, McCrone P, Seed PT, et al. Clinical- and cost-effectiveness of a nurse led self-management intervention to reduce emergency visits by people with epilepsy. PLoS One 2014;9:e90789.

40. Banerjee P, Hauser W. Incidence and Prevalence. In: Engel J, Pedley T, Aicardi J, eds. Epilepsy: a comprehensive textbook. Lippincott Williams \& Wilkins, 2007:45-56.

41. Reuber M, Hattingh L, Goulding PJ. Epileptological emergencies in accident and emergency: a survey at St James's university hospital, Leeds. Seizure 2000;9:216-20.

42. Reuber M, Torane P, Mack C. Do older adults have equitable access to specialist epilepsy services? Epilepsia 2010;51:2341-3.

43. Blank L, Baird W, Reuber M. Patient perceptions of the referral of older adults to an epilepsy clinic: do patients and professionals agree who should be referred to a specialist? Epilepsy \& behavior 2014;34:120-3.

44. Osborne A, Taylor L, Reuber M, et al. Pre-hospital care after a seizure: evidence base and United Kingdom management guidelines. Seizure 2015;24:82-7. 